\title{
Towards a History of Concepts of Light-Matter Coupling
}

\author{
V. V. Rumyantsev ${ }^{1}$, K. V. Gumennyk ${ }^{2}$ \\ ${ }^{1}$ Dept. of dynamical properties of complex systems, Galkin Institute for Physics \& Engineering, Donetsk, Ukraine \\ ${ }^{2}$ Dept. of electronic properties of metals, Galkin Institute for Physics \& Engineering, Donetsk, Ukraine
}

\section{Email address:}

vladimir.rumyantsev2011@yandex.ru (V. V. Rumyantsev), kgumennyk@gmail.com (K. V. Gumennyk)

\section{To cite this article:}

V. V. Rumyantsev, K. V. Gumennyk. Towards a History of Concepts of Light-Matter Coupling. Journal of Photonic Materials and Technology. Vol. 1, No. 1, 2015, pp. 1-9. doi: 10.11648/j.jmpt.20150101.11

\begin{abstract}
The article gives a succinct historical review of the evolution of physical concepts in photonics and light-matter coupling during the 20th century with special emphasis on noteworthy contributions made by a prominent Ukrainian theoretician Kirill Tolpygo, whose centenary will be celebrated next year. We dwell in detail on the history of elaboration of such key notions as various types of excitons, polaritons, spatial dispertion in crystals and others. Their correct understanding provides an indispensable basis for further progress in crystal optics.
\end{abstract}

Keywords: Photonics, Light-Mater Coupling, Crystal Lattice, Exciton, Polariton, Spatial Dispersion, K. B. Tolpygo

\section{Introduction}

Next year marks the centenary of a prominent Ukrainian theoretician, a Doctor of Theoretical and Mathematical Physics, a corresponding member of the Ukrainian Academy of Sciences Kirill Borisovich Tolpygo.

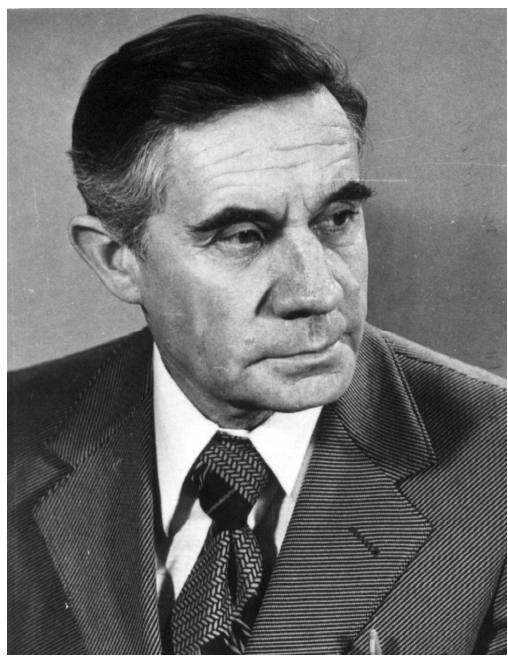

Fig. 1. Kirill Borisovich Tolpygo (03/05/1916 - 13/05/1994).

In 1949-1956 K.B. Tolpygo has pioneered a consistent development of adiabatic approximation in the dynamic theory of crystal lattices. He proposed a description, which permitted to account for deformation of electronic shells of ions and the concomitant effect of delay. The corresponding approach is known as Tolpygo's model or shell model. K.B. Tolpygo was the first to examine optical vibrations with due account for retardation effect. In doing so he obtained the mixed states of photons and phonons, which consequently were studied experimentally and have come to be known as polaritons. This has led to introduction of long-range Coulomb forces into the dynamics of homopolar and molecular crystals. Tolpygo's theory permitted to investigate an interaction between electrons and phonons of all braches and wave lengths and was successfully applied to polaritons, F-centers, and excitons in alkali halide crystals. Later this served as a basis for construction of a microscopic theory of local states of small-radius electrons.

It is due to point to Tolpygo's contribution to the manyelectron theory of valent crystals. He proposed an efficient method to account for many-electron correlation within the band theory and justified a quasi-molecular model for valent crystals. He also developed a novel interpretation of their optical absorption spectra based on the concept of Frenkel metastable exciton. K. B. Tolpygo has constructed a theory of defect formation under the light absorption from within the resonance band and gave a microscopic description of absorption of light waves incident on a semi-infinite crystal. Let us also mention his microscopic theory of Cherenkov radiation resulting from polariton generation by a fast electron field.

Of considerable interest are the works of K.B. Tolpygo devoted to kinetics and phenomenology of semiconductors, which covered the theory of thermionic emission, photoelectromotive force, $\mathrm{p}-\mathrm{n}$ transitions, surface phenomena 
etc. In addition to solid-state physics Tolpygo's research interests extended into the field of biophysics.

Making no pretence at exhaustiveness we essay below a survey of the currently topical field of photonics and lightmatter coupling, whose commencement and early development is to a large degree credited to K.B. Tolpygo.

\section{Excitons and Polaritons}

The microscopic theory of optical phenomena in crystals is closely related to the theory of excitons, which provides an appropriate tool for elucidation of the specifics of interaction between electromagnetic fields and crystals and for construction of the physical picture of energy transfer in crystalline media. It is well established that in a crystal, where the translation symmetry allows for excitation of any of its elementary cells (or any of the comprising structural units), the energy transfer of electronic excitations occurs due to the motion of quasi-particles called excitons. The concept of "exciton" was originally introduced into physics by the pioneering works of Ya.I. Frenkel [1, 2]. Among the first to consider excitonic states was also R. Peierls [3]. These works gave a theoretical explanation for the experimentally observed photoelectrically negative light absorption. Utilizing Heitler-London-Heisenberg method (HLH) in his study of electronic states [4] Ya.I. Frenkel demonstrated that the absence of photoconductivity under the light absorption by an electronic subsystem in an ideal crystal stems from excitations of crystalline structural units moving in waves (due to translation invariance) along a crystal. Such excited states were given the name of "Frenkel excitons".

Another excited no-current state of crystal electrons was considered by G. Wannier [5] and N. Mott [6]. It consists of an electron (in conductivity band) and a hole (in valence band) bound by coulombic forces. The center of inertia of such a hydrogen-like formation moves freely along a crystal. Wannier-Mott exciton motion can be described by the effective mass method [7]. Considering an excited state of a molecule as an electron-hole pair, the Frenkel exciton can be termed a small-radius exciton, while that of Wannier-Mott should be recognized as a big-radius one (with radius much bigger than the lattice constant). Excitations of the first type are mostly observed in molecular and alkali-halide crystals, whereas the second type is characteristic to semiconductors with narrow band gaps and large dielectric permittivity (there are exceptions to this rule though; see e.g. [8], where the properties of Frenkel excitons are discussed for $\mathrm{CuO}_{2}$ semiconductors).

A fairly exhaustive coverage of exciton theory and a detailed bibliography on the subject can be found in [9-15] Here we shall mention some of the works of principal importance.

An experimental evidence for the existence of WannierMott excitons was obtained by E.F. Gross and co-authors [16-18] by observing hydrogen-like spectra near the bandgap absorption edge. According to a well-known Rydberg formula the pattern of optical absorption lines in $\mathrm{CuO}_{2}$ stands in correspondence with excitonic levels (see e.g. [19]). In [20] authors reported on exciton absorption in GaAs. Ref. [21] brings an evidence for the motion of exciton in CdS crystalline lattice; Ref. [22] shows that CdS crystal anisotropy is manifested in anisotropy of excitonic states. Calculation of energy spectrum of excitons in alkali halide crystals was performed by T.A. Kudykina and K.B. Tolpygo [23], the corresponding calculation for CdS was given by S.A. Moskalenko and M.I. Shmiglyuk [24]. In the latter reference the authors point to the necessity of taking into account the splitting of excitonic terms resulting from the presence of identical ions in an elementary cell.

Frenkel excitons were studied in inert gas crystals [25-28]. Important investigations have also been carried out by A.S. Davydov and co-authors in a series of papers devoted to molecular excitons (see bibliography in [29]), where they for the first time considered the splitting of exciton states resulting from the presence of more than one molecule in an elementary cell. It was named Davydov splitting in contrast to Bethe splitting of degenerate atomic energy levels caused by intracrystalline field. Worthy of mentioning is also the paper of W. Heller and A. Marcus [30], which for the first time reports on splitting of energy levels of transverse and longitudinal excitons. A discontinuous character of exciton energy dependence on the wave vector $k$ for $k=0$ was established by S.I. Pekar in [31], where he took a proper account of long-range electric interactions between structural units in crystals. For specific models of excitons this result was verified in $[32,33]$.

S.I. Pekar generalized the concept of exciton [31] by calling "excitonic" an excited state in a non-conducting crystal characterized by a single continuous quantum number (quasi-impulse) with the rest of quantum numbers being discrete. Apart from the above discussed Frenkel and Wannier-Mott electronic excitons this general definition applies to optical phonons, molecular vibrons (electronoscillation excitations), spin waves etc.

The notion of exciton emerged from interpretation of light absorption bands in non-metallic crystals. According to theoretical models $[11,34]$ the pronounced resonance maxima of excitonic absorption spectra reflect a singleparticle character of light absorption, under which a photon in crystal transforms into a quasi-particle - an exciton. For small dissipation in the electronic subsystem and significant interaction between the said subsystem and the quanta of electromagnetic field the separate descriptions of the two subsystems lose their validity. In this situation an interaction between light and a crystal is described by Hamiltonian

$$
\hat{H}=\hat{H}_{e}+\hat{H}_{p}+\hat{H}_{e p},
$$

where $\hat{H}_{e}$ and $\hat{H}_{p}$ are, correspondingly, Hamiltonians of free excitons (dissipation processes, which affect optical properties in a crystal are neglected) and transverse photons (longitudinal Coulomb field is included in the term $\hat{H}_{e}$, since accounting for coulombic interaction should automatically 
yield excitonic states). Interaction Hamiltonian for excitons and photons has the form [34]:

$$
\begin{aligned}
& \hat{H}_{e p}=\hat{H}_{e p}^{(1)}+\hat{H}_{e p}^{(2)}, \\
& \hat{H}_{e p}^{(1)}=\sum_{\mathrm{k}, v, i} T_{i, v}(\mathrm{k})\left(a_{\mathrm{k} i}^{+}+a_{-\mathrm{k} i}\right)\left(B_{-\mathrm{k} v}-B_{\mathrm{k} v}^{+}\right), \\
& \hat{H}_{e p}^{(2)}=\sum_{\mathrm{k}, v, i} \frac{f_{v i}^{2}}{4 k c}\left(a_{\mathrm{k} i}^{+}+a_{-\mathrm{k} i}\right)\left(a_{\mathrm{k} i}+a_{-\mathrm{k} i}^{+}\right),
\end{aligned}
$$

where $a_{\mathrm{k} i}^{+}$and $a_{\mathrm{k} i}$ are creation and annihilation operators of a photon with wave vector $\mathrm{k}$ and polarization index $i, B_{\mathrm{k} v}^{+}$, $B_{\mathrm{k} v}$ are bosonic creation and annihilation operators of an exciton in the $v$-th band with wave vector $\mathrm{k}$ and energy $\omega(\mathrm{k})$; here $\hbar=1$. The explicit forms for the vertex part $T_{i v}(\mathrm{k})$ of interaction operator $\hat{H}_{e p}$ and the value $f_{v i}^{2}$ of the contribution of the given $v$-th transition into the square of plasma frequency in a crystal are given in [34].

Since Hamiltonian $\hat{H}$ is a bilinear combination of bosonic amplitudes, it can be reduced (through a canonic transformation) to a diagonal form, which yields the creation and annihilation operators of a new elementary excitation - a polariton [11]. An approach based on the concept of resonant transmutation of photons (in the infrared spectrum region) and excitons is called polaritonic (see e.g. [34]).

The problem of electromagnetic field oscillations "coupled" to crystal elementary excitations in the infrared spectrum region (where the most significant role is played by the optical branches of lattice oscillations) was for the first time solved on the basis of semi-classical approach by K.B. Tolpygo [35] and independently by M. Born and K. Huang $[36,37]$. Various solutions of this problem based on polaritonic approach were given by U. Fano [38], J. Hopfield [39], V.M. Agranovich [40] and A.A. Demidenko [41] (see also [42-45]). It will be recalled that the term "polariton" was introduced by J. Hopfield to denote the quantum of polarization oscillations of crystal structural units, which does not interact with a light wave. He proposed a crystal model, where atoms are replaced by uncoupled charged harmonic oscillators. In [39] J. Hopfield considered a combination of "polariton" and a vacuum photon, the so called crystal photon. It was for the latter that the term "polariton" was subsequently reserved.

There is an additional concept of exciton polariton introduced by S.I. Pekar to describe the mixed excitonelectromagnetic waves in crystals (see e.g. [46, 47]). The theory of exciton polaritons developed by S.I. Pekar and his co-authors proved extremely fruitful for solution of the problems of modern crystal optics with due account for interaction delay between crystal structural units as well as for spatial dispersion, long-range coulombic interactions and strong back action of crystal particles on electromagnetic waves.

When considering propagation of light in a crystal the effect of electromagnetic field on electrons and atomic nuclei can be regarded as a small quantity. The back action, however, of crystal charged particles on electromagnetic wave cannot be neglected. The energy of exciton-photon interaction is small as compared to the energy of electronnuclear subsystem, but isn't such when compared to the field energy in a crystal, especially in the excitonic absorption region.

In theories based on semiclassical approach this circumstance implies that polarization current in Maxwell equations cannot be regarded as a small quantity. However the same assumption is true for electromagnetic perturbation in Schrödinger's equations. Simultaneous solution of motion equations for the field and the crystal structural units yields a dispersion law, which characterizes a mixed "electronicmechanical" wave [46] and shows (see [37, 39, 48, 49]) that exciton energy is to a large extend comprised by mechanical energy of crystal nuclei and electromagnetic energy of the accompanying wave. The delayed character of particle interaction is accounted for in Maxwell equations. Passage to the limit $c \rightarrow \infty$ yields the results of a standard exciton theory based on the Schrödinger equation, which takes into account only coulombic interaction between crystal structural units and neglects the delay.

As is known [50], the delayed interaction between charged particles (in crystals and elsewhere) results from the exchange of virtual transversal photons. Neglecting the delay leads to a situation, where the lowest elementary excitations in crystals are excitons (in the sense defined in [48]) and transversal photons, whereas the genuine excitonic states are exciton polaritons.

The above conclusions are illustrated by Figs. 2, 3 (see [10, 37]). The dispersion law of uncoupled oscillatory systems (transversal photons and "mechanical" excitons) is depicted by dashed curves; solid curves correspond to coupled normal oscillations.

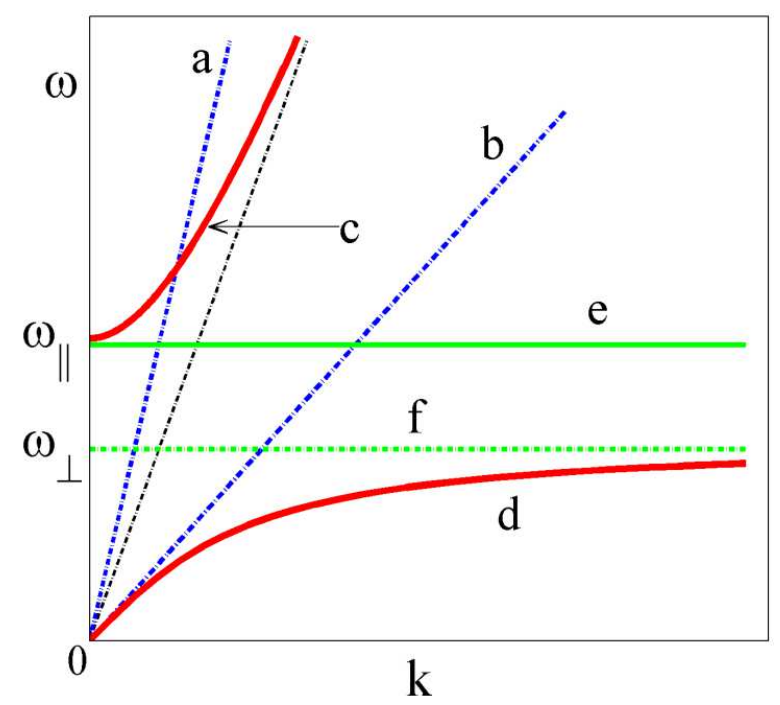

Fig. 2. Dependence of the frequency $\omega$ of normal waves (polaritons) propagating in an isotropic crystal on wave vector $k$. a) corresponds to light in a vacuum ( $\omega=c k), b)$ - to optical waves in a non-dispersive crystal, c) and d) - to coupled normal oscillations (polaritonic curves), e) and f) - to "mechanical" excitons (longitudinal and transversal, correspondingly) with delay neglected. 


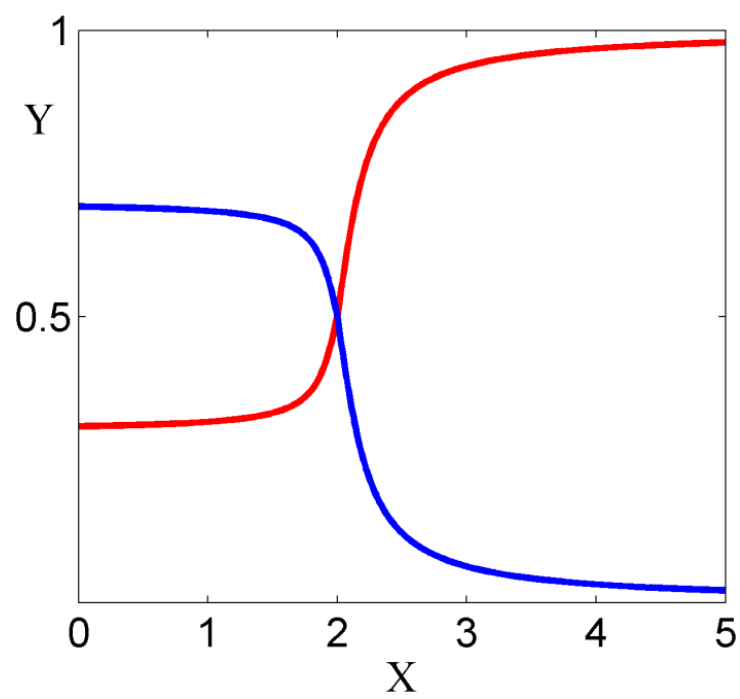

Fig. 3. Energy fraction $Y$ of "mechanical" excitons in the transversal normal oscillations of the "light in a crystal" system [37] ( $\left.X=c k / \omega_{\perp}\right)$.

\section{The Theory of Spatial Dispersion of Light in Crystals}

As has been justly observed by S.I. Pekar, when studying optical phenomena in crystals one should distinguish between "polaritonic" effects (which imply a strong interaction between crystal structural units and electromagnetic field) and spatial dispersion, i.e. the dependence of $\hat{\varepsilon}$ on $\omega$ and $\mathrm{k}$ along with its various manifestations such as additional light waves. Polaritonic effect has been in the focus of attention since the appearance of classical models of M. Born (1915, see e.g. [51]). At the same time the first significant manifestations of spatial dispersion in crystals were not revealed until 1957 [48].

Spatial dispersion is characterized by the quantity $a / \lambda$, where $a$ is the lattice constant and $\lambda$ is the typical scale of field variation (light wave length). In the optical frequency range $\left(a / \lambda \sim 10^{-3}\right.$, i.e. $\left.a / \lambda<<1\right)$ the spatial dispersion can be regarded as a small quantity except for certain cases. This circumstance accounts to a certain degree for a relatively late discovery of significant spatial dispersion effects. Not later than in the 19th century it has become clear though that in order to explain the natural activity (weak spatial dispersion) one should account for the dependence of dielectric permittivity $\hat{\varepsilon}$ on both the frequency $\omega$ and the wave vector $\mathrm{k}$. The term "spatial dispersion", which essentially states the dependence $\hat{\varepsilon}(\mathrm{k})$ was introduced by Hertzenshtein [53] in 1952.

Phenomenological description of electromagnetic wave propagation in a uniform medium is reduced to solution of the system of homogeneous equations with respect to Fourier components of the electric field strength $\mathrm{E}^{\omega, \mathrm{k}}$ :

$$
\left(k^{2} \delta_{i j}-k_{i} k_{j}-\frac{\omega^{2}}{c^{2}} \varepsilon_{i j}(\omega, \mathrm{k})\right) E_{j}^{\omega, \mathrm{k}}=0,
$$

where tensor $\hat{\varepsilon}(\omega, \mathrm{k})$ is a known function of $\omega$ and $\mathrm{k}$. The consistency condition for this system

$$
\operatorname{det}\left|k^{2} \delta_{i j}-k_{i} k_{j}-\frac{\omega^{2}}{c^{2}} \varepsilon_{i j}(\omega, \mathrm{k})\right|=0
$$

yields the dispersion law $\omega=\omega(\mathrm{k})$ of electromagnetic waves in a medium. Setting $\mathrm{k}=\frac{\omega}{c} n \mathrm{~s}$, where $n$ is the refractive index of light with frequency $\omega$ and propagation direction $\mathrm{S}$ one obtains from (4) the Fresnel equation for $n=n(\omega, \mathrm{s})$.

In the region of weak spatial dispersion tensor $\hat{\varepsilon}(\omega, \mathrm{k})$ and the inverse tensor $\hat{\varepsilon}^{-1}(\omega, \mathrm{k})$ can be expanded into Taylor series:

$$
\begin{aligned}
& \varepsilon_{i j}(\omega, \mathrm{k})=\varepsilon_{i j}(\omega)+i \gamma_{i j l}(\omega) k_{l}+\alpha_{i j l m}(\omega) k_{l} k_{m}+\ldots \\
& \varepsilon_{i j}^{-1}(\omega, \mathrm{k})=\varepsilon_{i j}^{-1}(\omega)+i \delta_{i j l}(\omega) k_{l}+\beta_{i j l m}(\omega) k_{i} k_{j}+\ldots
\end{aligned}
$$

Expansions (5) are equivalent throughout the spectrum except for the regions of broken smoothness of functions $\varepsilon_{i j}(\omega, \mathrm{k}), \varepsilon_{i j}^{-1}(\omega, \mathrm{k})$ (in the vicinity of their poles). In case one of components $\varepsilon_{i j}(\omega, \mathrm{k})$ drastically grows the first of expansions (5) becomes insufficient since its other terms become much smaller by comparison. $n(\omega)$ grows together with $\varepsilon_{i j}(\omega, \mathrm{k})$ and hence the role of spatial dispersion increases. In this situation spatial dispersion is taken into account by the second of expansions (5). Similar considerations apply to components $\varepsilon_{i j}^{-1}(\omega, \mathrm{k})$ in the vicinity of their poles. In (5) $\hat{\gamma}, \hat{\delta}$ are the tensors of optical activity (which do not appear in the case of non-gyrotropic medium), $\hat{\alpha}$ and $\hat{\beta}$ are fourth rank tensors, which define an additional crystal gyrotropy. Traditional crystal optics not only assumes the existence of equivalent expansions (5) (the choice between whom is made for the reasons of convenience) but also states the analyticity of their dependence on $\mathrm{k}$. This permits to fully utilize the symmetry of crystals in order to select non-zero components of tensors $\hat{\varepsilon}, \hat{\varepsilon}^{-1}$ (and, correspondingly, of $\hat{\alpha}, \hat{\beta}, \hat{\gamma}, \hat{\delta}$ ) as well as to establish their interdependence and ultimately to find the orientational dependence of optical effects (associated with expansion terms following the zeroth one).

In a broad spectrum region the effect of spatial dispersion gives no more than a minor correction to the already known optical characteristics of a medium. Even in traditional crystal optics however there are certain phenomena associated with spatial dispersion, such as gyrotropy (natural optical activity, NOA). Apart from NOA spatial dispersion plays a crucial role in propagation of longitudinal waves, which arise in plasma [55] but can also occur (subject to much bigger dissipation) in crystals. The dispersion law for longitudinal electromagnetic waves in an isotropic medium 
follows from the condition $\varepsilon_{||}(\omega, \mathrm{k})=0$, where $\varepsilon_{||}$is the coefficient entering the longitudinal part of tensor $\varepsilon(\omega, \mathrm{k})$ in an isotropic non-gyrotropic medium:

$$
\varepsilon_{i j}(\omega, \mathrm{k})=\left(\delta_{i j}-\frac{k_{i} k_{j}}{k^{2}}\right) \varepsilon_{\perp}(\omega,|\mathrm{k}|)+\frac{k_{i} k_{j}}{k^{2}} \varepsilon_{||}(\omega,|\mathrm{k}|) .
$$

Below we shall consider in more detail the specifics of spatial dispersion in cubic crystals with an inversion center. Tentatively it can be asserted that in this case the terms of order $a / \lambda$ in expansions (5) vanish, and hence the dispersion is characterized by the parameter $(a / \lambda)^{2}$. An observation on the necessity of higher approximations in the theory of double refraction in cubic crystals dates as far back as Lorentz's works on electronic theory of light dispersion. According to Hellwege [56] the physical meaning of approximation, which yields anisotropy of the order $(a / \lambda)^{2}$ consists in a proper account not only for dipole interaction with the matter, but also for quadruple transitions in crystals. Anisotropy in cubic crystals caused by spatial dispersion was pointed out by K.B. Tolpygo [57], S.I. Pekar [48], V.L. Ginzburg [54] and others. Experimental observation of optical anisotropy in the region of quadruple absorption line in cubic $\mathrm{Cu}_{2} \mathrm{O}$ crystals was performed by E.F. Gross and A.A. Kaplianski in 1960 [58].

\section{Crystal Optics in the Vicinity of Excitonic Transition Frequencies}

Parameter $a / \lambda=\frac{a}{\lambda_{0}} n$ (where $\lambda_{0}$ is the light wavelength in a vacuum) grows drastically in the vicinity of absorption (resonance) lines due to the growth of refraction index $n$, which leads to an increased role of spatial dispersion effects. A fundamentally new class of such effects was discovered by S.I. Pekar in 1957 [48], when he considered light waves in crystals with frequencies close to excitonic transitions. $\mathrm{He}$ demonstrated the existence of several waves with identical frequencies, polarizations and directions but different refraction indices - the so called additional light waves. This phenomenon should be distinguished from the double refraction; it is known to occur even in isotropically polarizable (cubic) crystals.

In his early works [48, 49, 59] S.I. Pekar used the following technique to find the dispersion law of exciton polaritons. In the context of Schrödinger's problem of particle motion the crystal Hamiltonian was supplemented by the energy of vortical constituent of the field and its interaction with the particles. The latter constituted a small external time-variant classical perturbation. The vortical part of the field was self-consistently found from the Maxwell equations whose right-hand sides contained charges and currents determined from Schrödinger's problem. The selfconsistent solution of such a problem yielded the dispersion law $\omega=\omega(\mathrm{k})$ of small oscillations in the conservative "crystal-electromagnetic field" system as well as the exciton polariton energy $u_{\mathrm{k}}=\hbar \omega(\mathrm{k})$.

Later S.I. Pekar gave another solution of this problem [46] based on techniques of the "conventional classical crystal optics". The macroscopic electromagnetic field (with wave length much bigger than the lattice constant) attending an exciton polariton was found from the Maxwell equation and the retardation of long-range interactions was taken into account forthwith. We recall that the energy of such light waves includes mechanical energy of particles (electrons and nuclei) comprising a crystal. The dispersion law obtained in [46] turned out to be identical to the previously found one. Such an approach therefore did not require the solution of an auxiliary Schrödinger's problem. It did however assume the knowledge of dielectric function $\varepsilon(\omega, \mathrm{k})$ which contained information on the motion of srystal structural units and was determined by the dispersion law of mechanical excitons $u_{e x}(\mathrm{k})$. For cubic crystals (with dissipation neglected) in the vicinity of $k=0$ holds the relation:

$$
\varepsilon(\omega, \mathrm{k})=\varepsilon_{0}+\frac{F^{i}}{\left(\frac{u_{e x}^{i}(0)}{\hbar}+\frac{\hbar^{2} k^{2}}{2 M_{e x}^{i}}\right)^{2}-\omega^{2}},
$$

where $M_{e x}^{i}$ is the effective mass of the $i$-th branch exciton, $\varepsilon_{0}$ is the contribution to dielectric permittivity of the rest of excited states in a crystal.

V.L. Ginzburg took an account of spatial dispersion (and obtained a "new wave") [54] by retaining an additional term dependent on $k$ in expansions (5). In [60] S.I. Pekar treated spatial dispersion by assuming both numerator and denominator in (7) dependent on $k$ (the former being decomposable into a series $F^{i}(\mathrm{k})=F_{1}^{i}+F_{2}^{i} k^{2}+\ldots$ and the latter containing a term proportional to $k^{2}$, which accounted for the motion of excitons). In all events [54, 48] the allowance for spatial dispersion leads to a higher degree of dispersion equation (similar to (4)) with respect to $n$ and hence to a higher number of roots. It is necessary therefore to carefully distinguish between those of additional roots, which correspond to genuine additional light and those corresponding to fictitious ones. This issue is throroughly discussed in the monograph by S.I. Pekar [60]. It is shown there that for a weak spatial dispersion $\left(\frac{a}{\lambda_{0}} n\right)^{2}$ all solutions of dispersion equation correspond to genuine light waves.

It is important to note that if the function $\varepsilon$ is decomposable in powers of $\delta=\frac{a}{\lambda_{0}} n[61]$ :

$$
\varepsilon(\omega, \delta)=\varepsilon_{0}+\varepsilon_{1}(\omega) \delta+\varepsilon_{2}(\omega) \delta^{2}+\ldots,
$$

then far from excitonic resonances $\varepsilon$ is a smooth function of $\delta$, i.e. 


$$
\frac{\varepsilon_{2}}{\varepsilon_{0}} \sim \frac{\varepsilon_{1}}{\varepsilon_{0}} \sim 1
$$

(normal spatial dispersion), whereas in the vicinity of the $i-$ th excitonic transition $\omega \rightarrow \omega_{i 0}=\frac{u_{e x}(0)}{\hbar}$ holds

$$
\frac{\varepsilon_{2}}{\varepsilon_{0}} \cup \frac{\varepsilon_{2}}{\varepsilon_{1}} \cup \frac{\varepsilon_{1}}{\varepsilon_{0}} \gg>1
$$

(anomalous dispersion).

Anomalous (in certain spectral regions) dependence of $\mathcal{E}$ on small parameter $\delta$ results in a qualitatively new finite effects such as Pekar's additional light waves (ALW) [60]. The occurrence of ALW at a normal incidence of light flux on a crystal leads to formation (in contrast with LambertBouguer law) of the interference pattern, since both the main and the additional waves have the same polarization $[62,63]$. Apart from experimental studies of interference reflection from CdSe thin plates [62, 63], the ALW were observed in $\mathrm{CdS}$ [64-68], and earlier in $\mathrm{Cu}_{2} \mathrm{O}$ [69].

Pekar's prediction of ALW [48] has created an unabating interest for experimental and theoretical investigations of spatial dispersion of dielectric permittivity and optical phenomena in the vicinity of excitonic resonances. Numerous works devoted to this subject have been published in 19701980 [70-77].

According to the modern understanding a crystal is constituted by a multitude of charged particles - electrons and atomic nuclei (for big kinetic energies one should also account for production and annihilation of electron-positron pairs). Due to electromagnetic interactions (which are mostly responsible for physical and chemical properties of crystals) particles execute a complex motion, resulting in a certain ordered structure of crystalline medium. In its general formulation the problem of dynamics of a "crystalelectromagnetic field" system is reduced to a self-consistent solution of equations, defining the evolution of crystal structural units under the action of field in combination with field equations. The necessary calculations are usually performed with the use of a certain approximation, which is chosen individually for each particular case. This choice depends on crystal model as well as on the nature of excitations involved. For instance, in the optics of ionic crystals in the infrared spectrum region the major role is played by optical branches of lattice oscillations [35-37], while in the higher frequency region this role belongs to electronic-type excitations [10,15].

In order to obtain the dispersion law of the "light in a crystal" system the latter is usually subdivided into a "crystal" itself and "electromagnetic field" with interaction Lagrangian written in the linear approximation as $\frac{1}{c} \mathrm{j} \cdot \mathrm{A}-e \varphi$

(where $\varphi$ and $\mathrm{A}$ are the scalar and vector potentials of the field, respectively). Genuine excited states of such a system (exciton polaritons) are determined via diagonalization of the full Hamiltonian (see e.g. [34]). Hamiltonian of the "crystal" contains either short range forces acting between electrons of different atoms (such excitations are called "mechanical excitons") or instantaneous coulombic interaction between electrons (coulombic excitons) while interaction term contains only the transverse field component. An alternative (phenomenological) approach in crystal optics involves the choice of specific form of dielectric response $\hat{\varepsilon}$ (individual for each particular crystal model) and then the dispersion law is obtained from an equation similar to (4).

K.B. Tolpygo, while not denying the results obtained by traditional methods, pointed in [76] to the difficulties of constructing the crystal optics in the vicinity of exciton formation frequency. The traditional approach has the following shortcomings. 1. Noting that the most popular choice for $\hat{\varepsilon}$ is Eq. (7) the authors of [77] demonstrate that it leads to violation of the causality principle (the response comes ahead of the light signal at short distances off the perturbation source), which results from the "coercive" inclusion in (7) of the dispersion law of mechanical excitons (corresponding to an instantaneous interaction between the structural units of a crystal). 2. According to [76, 77] the concept of crystal, where the only acting forces are either short-range forces or instantaneous coulombic interactions is glaringly artificial. An arbitrary subdivision of the field into longitudinal and transverse components is not relativistic invariant, which makes coulombic excitons look different in various reference systems. Also in the region of significant spatial dispersion the perturbation cannot be reduced to a transverse field, since in the said region the field necessarily possesses a certain longitudinal component. 3. Treatment of electromagnetic field in a crystal as an "external perturbation" acting on a given medium is contradictory. The genuine external field is an incident wave coming out of vacuum whose propagation velocity is $c$ (not $c / n)$. As for the transverse field $\left(-\frac{1}{c} \mathrm{~A}\right)$ it includes all the fields created by virtually excited atoms. In a crystal each structural unit is subject both to external and ambient fields, which requires a careful exclusion of "self-actions" and an accurate finding of the perturbation acting on structural units. Considering an excited state of the entire crystal one risks accounting for certain interactions more than one time.

Hence for the frequently used crystal model consisting of weakly interacting structural units it is more suitable to use the approach described in $[75,76]$, where the light propagation in a crystal is treated by considering molecules and the field surrounded by vacuum. This eliminates contradictions, which arise when we treat a crystal on one hand as an object under the action of the field and on the other hand as a medium where the said field propagates. This also removes the shortcomings stemming from the necessity to relate one of the field components (e.g. a longitudinal one) to interatomic interactions (in order to be able to construct quantum states of the crystal) while considering the other as "light". Light propagation in a crystal can then be interpreted as a retarded excitation transfer of its molecules. 


\section{Conclusion}

Results in crystal optics obtained during the past fifty years provide a solid foundation for the progress of modern photonics. Concepts developed in the physics of crystalline solids can potentially be applied to photonic supercrystals. While the theory of impurity bands and excitons in semiconductor crystals has been constructed in 1970-1980, an analogous theory for photonic crystals is yet to be completed. Recent experiments and theoretical investigations reveal an intense interest for polaritonic structures and systems of coupled microresonators [78,79], whose applications include fabrication of clockworks of unprecedented accuracy [80] as well as of the sources of coherent irradiation. There has been a significant advance in the photonics of imperfect structures. A number of our recent works have been devoted to optical activity of imperfect photonic crystals [81] and to dispersion of exciton-like electromagnetic excitations in non-ideal lattices of coupled microresonators [82-84]. Such investigations provide the necessary theoretical background for manufacturing of composite structures with controllable propagation of electromagnetic waves.

\section{References}

[1] J. Frenkel, "On the transformation of light into heat in solids", Phys. Rev., vol. 37, pp. 1276-1294, January 1931.

[2] R. Peierls, „Zur Theorie der Absorptionsspektren fester Körper“, Ann. Phys., vol. 13, pp. 905-952, 1932.

[3] W. Heitler and F. London, "Wechselwirkung neutraler Atome und homöopolare Bindung nach der Quantenmechanik", Z. Phys., vol. 44, pp. 455-472, June 1927.

[4] W. Heisenberg, "Zur Theorie des Ferromagnetismus", Z. Phys., vol. 49, pp. 619-636, May 1928.

[5] G.H. Wannier, "The structure of electronic excitation levels in insulating crystals", Phys. Rev. vol. 52, pp. 191-197, August 1937.

[6] N.F. Mott, "Conduction in polar crystals. II. The conduction band and ultra-violet absorption of alkali-halide crystals", Trans. Faraday Soc., vol. 34, pp. 500-506, January 1938.

[7] S. Pekar, "The method of effective electron mass in crystals", Zh. Eksp. Teor. Fiz., vol. 16, p. 933, 1946.

[8] N.V. Starostin, "Energy spectrum of Frenkel excitons in $\mathrm{Cu} 2 \mathrm{O}$ crystal”, Opt. Spectr., vol. 22, pp. 646-652, April 1967.

[9] C. Kittel, Quantum Theory of Solids. New York: Wiley, 1987.

[10] R.S. Knox, Theory of Excitons. New York: Academic Press, Inc., 1963.

[11] V.M. Agranovich, Theory of Excitons. Moscow: Nauka, 1968.

[12] A.S. Davydov, Theory of Molecular Excitons. New York: McGraw-Hill, 1962.

[13] A.S. Davydov, The Theory Of Light Absorption In Molecular Crystals. Kiev: Izd. Akademii nauk USSR, 1951.
[14] A.S. Davydov, "Theory of excitons and solitons in molecular systems (review)", J. Appl. Spectros., vol. 33, pp. 1029-1047, October 1980.

[15] H. Haken, "Die Theorie des Exzitons im festen Körper", Fortschr. Phys., vol. 6, pp. 271-334, 1958.

[16] E.F. Gross and M.A. Yacobson, "The fine structure of the intrinsic absorption edge in a CdS crystal”, Dokl. Akad. Nauk. SSSR, vol. 102, pp. 485-488, 1955.

[17] D. Fishman, Excitons in cuprous oxide, Doctoral thesis, University of Groningen, 2008.

[18] E.F. Gross, "Excitons and their motion in crystal lattices", Sov. Phys. Usp., vol. 5, pp. 195-218, 1962.

[19] P.W. Baumeister, "Optical absorption of cuprous oxide”, Phys. Rev., vol. 121, pp. 359-363, January 1961.

[20] M.D. Sturge, "Optical absorption of gallium arsenide between 0.5 and 2.75ev", Phys. Rev. vol. 127, pp. 768-773, August 1962.

[21] D.G. Thomas and J.J. Hopfield, "A magneto-stark effect and exciton motion in CdS", Phys. Rev., vol. 124, pp. 657-665, November 1961.

[22] M. S. Brodin and M. I. Strashnikova, "Optical properties of a CdS single crystal well inside the fundamental absorption region and the structure of energy bands (UV absorption and dispersion of cadmium sulfide crystals and energy band structure)", Sov. Phys. Sol. St., vol. 8, pp. 549-551, 1966.

[23] T.A. Kudykina and K.B. Tolpygo, "Exciton of high symmetry in alkali-halide crystals", Fiz. Tverd. Tela, vol. 14, pp. 626$628,1972$.

[24] S.A. Moskalenko and M.I. Shmiglyuk, "On the energy spectrum of excitons in CdS-type crystals", Sov. Phys. Sol. St., vol. 6 , pp. 2831-2833, 1964.

[25] G. Baldini, "Ultraviolet absorption of solid argon, krypton and xenon”, Phys. Rev., vol. 128, pp. 1562-1567, November 1962.

[26] I.T. Steinberger, C. Atluri and O. Schnepp, "Optical constants of solid xenon in the vacuum-uv region", J. Chem. Phys., vol. 52, pp. 2723-2729, March 1970.

[27] I.Ya. Fugol', E.V. Savchenko and A.G. Belov, "Luminescence of solid neon”, JETP Lett., vol. 16, pp. 172-174, 1972.

[28] A.G. Belov, I.Ya. Fugol' and E.V. Savchemko, "Cathodoluminescense of rare-gas solid solution", Sol. St. Comm., vol. 12, pp. 1-4, January 1973.

[29] A.S. Davydov, Theory of Solid State. Moscow: Izd. Nauka, 1976.

[30] W.R. Heller and A.A. Marcus, "A note on the propagation of excitation in an idealized crystal", Phys. Rev., vol. 84, pp. 809-813, November 1951.

[31] S.I. Pekar, "Energy of excitons with limitingly small quasiimpulses", Zh. Eksp. Teor. Fiz., vol. 35, pp. 522-524, 1958.

[32] D. Fox and S. Vatsiv, "Anisotropic effects in optical excitation of excitons in molecular crystals", Phys. Rev., vol. 108, pp. 938-945, November 1957.

[33] V.L. Bonch-Bruevich and Sh.M. Kogan, "The theory of electron plasma in semiconductors", Sov. Phys. Sol. St., vol. 1, 1960. 
[34] M.S. Brodin, E.N. Myasnikov and S.V. Marisova, Polaritons in crystal optics. Kiev: Naukova Dumka, 1984.

[35] K.B. Tolpygo, "Physical properties of the salt lattice constructed from deforming ions", Zh. Eksp. Teor. Fiz., vol. 20, pp. 497-509, 1950.

[36] K. Huang, "On the interaction between the radiation field and ionic crystals", Proc. Roy. Soc. Ser. A, vol. 208, pp. 352-365, September 1951.

[37] M. Born and K. Huang, Dynamical Theory of Crystal Lattices. Oxford: Clarendon Press, 1998.

[38] U. Fano, "Atomic theory of electromagnetic interactions in dense materials", Phys. Rev., vol. 103, pp. 1202-1218, September 1956.

[39] J.J. Hopfield, "Theory of the contribution of excitons to the complex dielectric constant of crystals", Phys. Rev., vol. 112, pp. 1555-1567, December 1958.

[40] V. M. Agranovich, "Dispersion of electromagnetic waves in crystals", Sov. Phys. JETP, vol. 10, pp. 307-313, 1960.

[41] A.A. Demidenko, "Microtheory of the Frenkel exciton with and without delay", Sov. Phys. Sol. St., vol. 3, pp. 869-879, 1961.

[42] M. Artoni and J.L. Birman, "Quantum-optical properties of yolariton waves", Phys.Rev. B, vol. 44, pp. 3736-3756, August 1991.

[43] M. Artoni and J.L. Birman, "Stationary and time-dependent quantum effects in polaritons", Laser Phys., vol. 5, pp. $584-$ 589, 1995.

[44] G. Qin, K.L. Wang and Z.D. Wang, "Exact analytical solution of a polariton model: undetermined coefficient approach", Phys.Rev. A, vol. 66, pp. 025804 1-4, August 2002.

[45] F. Bassani, "Polaritons" in Electronic excitations in organic based nanostructures, V.M. Agranovich and G.F. Bassani, Eds. Amsterdam: Academic Press, 2003, pp. 129-183.

[46] S.I. Pekar, "Identification of excitons with light waves in a crystal and the macroscopic theory of excitons with and without account of retardation", Sov. Phys. JETP, vol. 11, pp. 1286-1293, 1960.

[47] G. F. Glinskii and Z. G. Koinov, "Polariton theory of light propagation in crystals I. connection between the microscopic theory of exciton-phonon polaritons and Maxwell equations". Phys. Stat. Sol. (b), vol. 155, pp. 501-512, October 1989.

[48] S.I. Pekar, "The theory of electromagnetic waves in a crystal in which excitons are produced", Sov. Phys. JETP, vol. 6, p. $785,1958$.

[49] S.I. Pekar, "Theory of the absorption of light and dispersion in crystals", Zh. Eksp. Teor. Fiz., vol. 36, p. 451, 1959.

[50] V.B Berestetskii, E.M. Lifshitz and P. Pitaevski, Quantum Electrodynamics. Oxford: Butterworth-Heinemann, 1982.

[51] F. De Martini, "Nonlinear-optics of bulk-polaritons", in Polaritons: Proceedings of the first Taormina research conference on the structure of matter, October 2-6, 1972, Taormina, Italy and reprints of selected key papers from the literature, E. Burstein and F. De Martini, Eds. New York: Pergamon Press, 1974.
[52] A.A. Rukhadze and V.P. Silin, "Electrodynamics of media with spatial dispersion", Sov. Phys. Usp., vol. 4, pp. 459-484, 1961.

[53] M.E. Gertsenshtein, "Longitudinal waves in an ionized medium (plasma)”, Zh. Eksp. Teor. Fiz., vol. 22, pp. 303-309, 1952.

[54] V.L. Ginzburg, "Electromagnetic waves in isotropic and crystalline media with account of spatial dispersion of the dielectric constant", Sov. Phys. JETP, vol. 34, pp. 1593-1604, 1958.

[55] B.N. Gershman, "Note on waves in a homogeneous magnetoactive plasma”, Sov. Phys. JETP, vol. 4, 1957.

[56] K.H. Hellwege, "Optische anisotropie kubischer kristalle bei quadrupolstrahlung”, Z. Phys., vol. 129, pp. 626-641, 1951.

[57] K.B. Tolpygo, "The present state of the theory of polarization of ideal ionic and valence crystals", Sov. Phys. Usp., vol. 4, pp. 485-497, 1961.

[58] E. F. Gross and A.A. Kaplianskii, "Optical anisotropy of cubic crystals produced by dispersion in a space-quadrupole excitonic absorption of light in cuprous oxide", Dokl. Akad. Nauk SSSR, vol. 132, pp. 98-101, 1960.

[59] S.I. Pekar, "Dispersion of light in the exciton absorption region of crystals", Sov. Phys. JETP, vol. 7, pp. 813-822, 1958.

[60] S.I. Pekar, Crystal Optics and Additional Light Waves. Menlo Park, CA: Benjamin-Cummings, 1983.

[61] V.N. Piskovoy, The theory of optical and acoustoelectric phenomena associated with anomalies in the spatial dispersion as well as in the field and deformational dependences of dielectric responses of crystals, Habilit. Thesis, ISP NAS USSR, Kiev, 1984.

[62] V.A. Kiselev, B.S. Razbirin and I.N. Ural'tsev, "Interference states of optical excitons. Observation of additional waves", Sov. Phys. JETP Lett., vol. 18, pp. 296-298, 1973.

[63] V.A. Kiselev, B.S. Razbirin and I.N. Uraltsev, "Additional waves and Fabry-Perot interference of photoexcitons (polaritons) in thin II-VI crystals", Phys. St. Sol., vol. B72, pp. 161-172, November 1975.

[64] M.I. Strashnikova and A.T. Rudchik, "On shape of exciton absorption bands in CdS monocrystals at $4.2 \mathrm{~K}$ ", Fiz. Tverd. Tela, vol. 14, pp. 984-988, 1972.

[65] M. S. Brodin, N. A. Davydova and M. I. Strashnikova, "Anomalous dispersion of a CdS single crystal in the excitonabsorption region", Pis'ma Zh. Eksp. Teor. Fiz., vol. 19, pp. $567-571,1974$.

[66] M.I. Strashnikova, "Failure of classical Fresnel relationships in the exciton absorption region", Sov. Phys. Sol. St., vol. 17, pp. 467-470, 1975.

[67] S.I. Pekar and M.I. Strashnikova, "Spatial dispersion and additional light waves in the region of exciton absorption in CdS”, Zh. Eksp. Teor. Fiz., vol. 68, pp. 2047-2054, 1975.

[68] J. Braser, M. Rosenzweig, R. Braser, M. Richard and E. Birkcht, "A quantitative study of excitonic polariton reflectance in CdS", Phys. St. Sol., vol. B90, pp. 77-91, November 1978. 
[69] I.S. Gorban and V.B. Timofeev, "Complex refraction in single cuprous oxide crystals”, Dokl. Akad. Nauk SSSR, vol. 140, pp 791-793, 1961.

[70] S. I. Pekar and B. E. Tsekvava, "The dispersion of light in exciton absorption regions in cubic crystals as affected by the anisotropy of the effective mass of the exciton", Sov. Phys. Sol. St., vol. 2, pp. 242-251, 1960.

[71] G.S. Agarwal, D.N. Pattanayak, and E. Wolf, "Structure of the electromagnetic field in a spatially dispersive medium", Phys. Rev. Lett., vol. 27, pp. 1022-1025, October 1971.

[72] P.R. Rimbey, "Polariton Green's functions for semi-infinite spatially dispersive media with applications to molerular crystal reflectivity spectra", J. Chem. Phys., vol. 67, pp. 698709, July 1977.

[73] A.A. Demidenko, S.I. Pekar and E. Tsekvava, "Contribution to the theory of spatial dispersion and auxiliary light waves in the exciton absorption region", Sov. Phys. JETP, vol. 49, pp. 734-740, April 1979.

[74] E. Rashba and M. Sterge, Excitons. Amsterdam: NorthHolland Publ., 1982.

[75] V.V. Rumyantsev and, K.B. Tolpygo, "Light propagation in a crystal in the vicinity of a quadrupolar permitted transition", Ukr. Phys. J., vol. 30, pp. 699-707, 1985.

[76] K.B. Tolpygo, "Propagation of light in a crystal as a retarded excitation transfer of its atoms", Ukr. Fiz. Zh., vol. 31, pp. 178-187, 1986.

[77] A.A. Borgardt and K.B. Tolpygo, "Space-time dispersion, dielectric permittivity and the causality principle", Ukr. Fiz. Zh., vol. 23, pp. 607-615, 1978.
[78] P. Del'Haye, A. Schliesser, O. Arcizet, T. Wilken, R. Holzwarth and T. J. Kippenberg, "Optical frequency comb generation from a monolithic microresonator", Nature, vol. 450, pp. 1214-1217, December 2007.

[79] D. Hou, B. Ning, J. Wu, Z. Wang and J. Zhao, "Demonstration of a stable erbium-fiber-laser-based frequency comb based on a single rubidium atomic resonator", Appl. Phys. Lett., vol. 102, pp. 151104 1-4, April 2013.

[80] S.B. Papp, K. Beha, P. Del'Haye, F. Quinlan, H. Lee, K.J. Vahala and S.A. Diddams, "Microresonator frequency comb optical clock", Optica, vol. 1, pp.10-14, July 2014.

[81] E.S. Sedov, A.P. Alodjants, S. M. Arakelian, Y.Y. Lin and R.-K Lee, "Nonlinear properties and stabilities of polaritonic crystals beyond the low-excitation-density limit", Phys. Rev. A, vol. 84, pp. 013813, July 2011.

[82] V.V. Rumyantsev, S.A. Fedorov, K.V. Gumennyk and M.V. Proskurenko, "Peculiarities of propagation of electromagnetic excitation through a nonideal gyrotropic photonic crystal", Physica B, vol. 442, pp. 57-59, June 2014.

[83] V.V. Rumyantsev, S.A. Fedorov, K.V. Gumennyk, M.V. Sychanova and A.V. Kavokin, "Exciton-like electromagnetic excitations in non-ideal microcavity supercrystals", Nature. Sci. Rep., vol. 4, art. No. 6945, November 2014.

[84] V.V. Rumyantsev, S.A. Fedorov, K.V. Gumennyk and M.V. Sychanova, "Dispersion characteristics of electromagnetic excitations in a disordered 1D lattice of coupled microresonators", Physica B, vol. 461, pp. 32-37, March 2015. 